\title{
Complex regulation of neutrophil-derived MMP-9 secretion in central nervous system tuberculosis
}

\author{
Catherine W. M. Ong ${ }^{1,2}$, Przemyslaw J. Pabisiak', Sara Brilha ${ }^{1}$, Poonam Singh ${ }^{3}$, Federico Roncaroli ${ }^{4}$, \\ Paul T. Elkington ${ }^{1,5}$ and Jon S. Friedland ${ }^{1 *}$
}

\begin{abstract}
Background: Central nervous system tuberculosis (CNS-TB) may be fatal even with treatment. Neutrophils are the key mediators of TB immunopathology, and raised CSF matrix metalloproteinase-9 (MMP-9) which correlates to neutrophil count in CNS-TB is associated with neurological deficit and death. The mechanisms by which neutrophils drive TB-associated CNS matrix destruction are not clearly defined.

Methods: Human brain biopsies with histologically proven CNS-TB were stained for neutrophils, neutrophil elastase, and MMP-9. Neutrophil MMP-9 secretion and gene expression were analyzed using Luminex and real-time PCR. Type IV collagen degradation was evaluated using confocal microscopy and quantitative fluorescent assays. Intracellular signaling pathways were investigated by immunoblotting and chemical inhibitors.

Results: MMP-9-expressing neutrophils were present in tuberculous granulomas in CNS-TB and neutrophil-derived MMP-9 secretion was upregulated by Mycobacterium tuberculosis (M.tb). Concurrent direct stimulation by M.tb and activation via monocyte-dependent networks had an additive effect on neutrophil MMP-9 secretion. Destruction of type IV collagen, a key component of the blood-brain barrier, was inhibited by neutralizing neutrophil MMP-9. Monocyte-neutrophil networks driving MMP-9 secretion in TB were regulated by MAP-kinase and Akt-PI 3 kinase pathways and the transcription factor NF-kB. TNFa neutralization suppressed MMP-9 secretion to baseline while dexamethasone did not.
\end{abstract}

Conclusions: Multiple signaling paths regulate neutrophil-derived MMP-9 secretion, which is increased in CNS-TB. These paths may be better targets for host-directed therapies than steroids currently used in CNS-TB.

Keywords: Tuberculosis, Immunopathology, Neutrophils, Matrix metalloproteinase

\section{Background}

Despite treatment, central nervous system tuberculosis (CNS-TB) often results in severe neurological deficit and frequent death. Dexamethasone, a recommended adjunct to CNS-TB treatment, improves mortality but does not affect long-term neurological outcome $[1,2]$. This has been attributed to innate host factors such as the $\mathrm{LTA}_{4}$ genotype [3], underscoring the role of host-driven immunopathology in this devastating infection. Newer

\footnotetext{
* Correspondence: j.friedland@imperial.ac.uk

${ }^{1}$ Section of Infectious Diseases and Immunity, Hammersmith Campus, Imperial College London, 8th floor Commonwealth Building, Du Cane Road, London W12 ONN, UK

Full list of author information is available at the end of the article
}

interventions for CNS-TB are urgently needed to improve both morbidity and mortality, particularly in the era of rising drug resistance. Host-directed therapies may be an attractive therapeutic strategy [4].

Neutrophils are emerging mediators of TB pathology. These early sentinels to Mycobacterium tuberculosis infection play the key roles in TB inflammation $[5,6]$. Raised neutrophils are present in CNS-TB in the setting of both HIV-negative and HIV-associated immune reconstitution inflammatory syndrome and increased neutrophil-associated mediators such as S100A calciumbinding protein correspond to the degree of inflammation [7-9]. However, the mechanisms by which 
neutrophils cause neuroinflammation in CNS-TB are not defined.

The development of severe neurological deficits may be caused by local CNS tissue destruction. Tissue damage may be driven by the host immune cells recruited to the CNS such as neutrophils and macrophages $[10,11]$, following disruption to the blood-brain barrier (BBB). These cells secrete matrix metalloproteinases (MMPs), zinc-containing proteases which degrade extracellular matrix fibrils crucial for the integrity of the BBB [12]. MMPs are inhibited by specific tissue inhibitors of metalloproteinases (TIMPs). We and others found increased MMP expression in CNS-TB and raised MMP concentrations were associated with neurological deficit and death [7, 8, 13]. In addition, mediators including $\mathrm{TNF} \alpha$ which is the key in the defense against mycobacteria and whose blockade leads to reactivation of TB [14, 15], drive MMP secretion from the host cells including the neutrophils and epithelial cells and may have a role in CNS-TB immunopathology [16, 17]. Investigating mechanisms by which MMPs result in tissue damage may be the key in understanding the pathogenesis of CNS neuroinflammation caused by agents such as M.tb.

We hypothesized that neutrophils drive matrix destruction in CNS-TB. As humans are the primary host of $M . t b$, we examined the brain biopsies of patients with proven CNS-TB and investigated our findings in a human cellular model. Neutrophils expressing MMP-9 are present in CNS tuberculous granulomas, and M.tb infection increased neutrophil MMP-9 secretion and gene expression. Neutrophil-derived MMP-9 was functionally active and caused type IV collagen destruction, which was reversed by neutralizing MMP-9. We demonstrate that mitogen activated protein-kinase (MAP-kinase) and the phosphoinositide-3 $\left(\mathrm{PI}_{3}\right)$ kinase-Akt pathways regulated neutrophil MMP-9 secretion in monocyte-dependent intercellular networks. Neutralizing TNF $\alpha$ suppressed neutrophil MMP-9 to baseline, while dexamethasone did not, which may partly explain the limited benefit of steroids in patients. Taken together, our findings suggest that hostdirected therapy targeting MMP-9 secretion may have a potential to limit immunopathology in CNS-TB.

\section{Methods}

\section{Reagents and antibodies}

Dexamethasone was from Sigma. Helenalin and SC-514 were from Merck Biochemicals. SB203580, PD98059, and LY294002 were from Enzo Life Sciences. Goat antihuman TNFa was from PeproTech. Mouse anti-human MMP-9, rabbit anti-M.tb, and goat anti-rabbit IgG Cy5 were from Abcam. Rabbit anti-human phospho-Akt, total-Akt, phospho-p38, total-p38, phospho-ERK, totalERK, phospho-JNK, total-JNK, and goat anti-rabbit HRP linked were from Cell Signaling Technology. Rabbit anti-human neutrophil elastase was from Dako, and mouse anti-human MMP-9 was from Millipore.

\section{Recruitment of patients and controls Immunohistochemistry}

The paraffin blocks of five surgical samples from immunocompetent patients with CNS M.tb infection were retrieved from the files of the Department of Histopathology at Imperial College Healthcare Trust, London. All specimens contained leptomeninges, cortex, and subcortical white matter and showed typical necrotizing granulomas and acid-fast bacilli identified with the Ziehl-Neelsen stain. Sections of caecal appendix with acute inflammation were used as positive control for MMP-9 immunoreactivity. To prove the specificity of primary antibodies directed against MMP-9 and elastases, we used sections from the frontal lobe of five post-mortem brain with only mild aging-related changes. The appendix and brains were also retrieved from the files of the Department of Histopathology at Imperial College. Samples were annonymized for the purpose of this study. The cases were investigated using immunoperoxidase immunohistochemistry with antibodies directed against MMP-9 (Abcam; 1: 200) and neutrophil elastase (Dako, clone NP57; 1/100). Immunostains with omission of the primary antibody were performed as negative controls. Five-micron sections were cut from each block, dewaxed in xylene, and rehydrated in decreasing alcohols to distilled water. Endogenous peroxidase activity was blocked in $0.3 \%$ hydrogen peroxide in methanol for $30 \mathrm{~min}$. For antigen retrieval, sections were steamed for $20 \mathrm{~min}$ in $0.01 \mathrm{M}$ citrate, $\mathrm{pH} 6.5$, and then gently cooled in water. In order to block nonspecific binding of the primary antibody, sections were incubated with $10 \%$ normal goat serum for 10 min (Vector Laboratories, Burlingame, California). The primary antibodies were applied overnight at $4{ }^{\circ} \mathrm{C}$. After incubation, they were washed for three times in PBS for 10 min each. Staining was visualized using the VECTASTAIN Elite $A B C$ Kit (Vector Laboratories) following the manufacturer instruc-

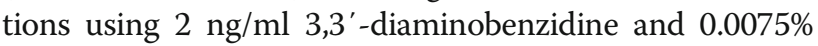
hydrogen peroxide in PBS as chromogen.

\section{M.tb culture}

M.tb H37Rv was cultured in supplemented Middlebrook 7H9 medium (BD Biosciences). For infection experiments, mycobacteria were used at mid-logarithmic growth at an optical density of 0.60 (Biowave cell density meter; WPA).

\section{Cell culture and stimulation}

The whole blood from healthy volunteers were drawn in preservative-free heparin and mixed with equal volumes of $3 \%$ dextran saline to remove erythrocytes. Neutrophils 
were isolated from the resulting cell suspension using Ficoll-Paque density centrifugation and three rounds of hypotonic lysis. Neutrophil purity was over $95 \%$ by FACS and viability $>99 \%$ by trypan blue assay. In some experiments, neutrophils were pre-incubated with specific inhibitors/agents as indicated for $30 \mathrm{~min}$ unless otherwise stated. In all experiments involving live M.tb H37Rv, tissue culture medium was sterile filtered through $0.2 \mu \mathrm{m}$ Anopore membranes (Millipore) before removing from the containment level 3 tuberculosis laboratory. All experiments were performed using $4 \mathrm{~h}$ incubations unless otherwise stated.

Primary human blood monocytes were prepared from donor leukocyte cones from healthy donors (National Blood Transfusion Service, UK). After density gradient centrifugation (Ficoll-Paque) followed by adhesion purification, monocyte purity was over $95 \%$ by FACS analysis. Monocytes were infected with M.tb at a multiplicity of infection (MOI) of 1 . After incubation at $37{ }^{\circ} \mathrm{C}$ for $24 \mathrm{~h}$, conditioned medium was harvested and was termed CoMTB. Media from uninfected monocytes were termed CoMCont.

\section{ELISAs for TIMP-1/2, MPO, and NGAL}

TIMP-1 and -2 concentrations were measured using the Duoset ELISA Development System (R\&D Systems) and detected a minimum of $31.2 \mathrm{pg} / \mathrm{ml}$ for both. Myeloperoxidase (MPO) was measured using the human MPO Quantikine ELISA Kit (R\&D Systems) which had a minimum detection limit of $0.1 \mathrm{ng} / \mathrm{ml}$. Neutrophil gelatinaseassociated lipocalin (NGAL) was measured using the human NGAL ELISA Kit (BioPorto Diagnostics) which had minimum detection limit of $1.6 \mathrm{pg} / \mathrm{ml}$.

\section{Luminex array}

MMP-8 and -9 concentrations were analyzed by Fluorokine MultiAnalyte Profiling Kit according to manufacturer's protocol (R\&D Systems) on the Luminex 200 platform (Bio-Rad). The minimum level of detection for MMP-8 and -9 was 110 and 65 pg/ml, respectively.

\section{DQ collagen degradation assay}

Type IV collagen degradation was assessed using the EnzChek ${ }^{\circledR}$ Gelatinase/Collagenase Assay kit (Molecular Probes). Samples were activated with $2 \mathrm{mM}$ of 4 -aminophenyl mercuric acetate (APMA) for $1 \mathrm{~h}$ at $37^{\circ} \mathrm{C}$. Eighty microliters of reaction buffer or inhibitor (mouse antihuman MMP-9) were added with $20 \mu \mathrm{L}$ of DQ collagen (Invitrogen) at a final concentration of $25 \mu \mathrm{g} / \mathrm{ml}$. The activated samples were subsequently added, and activity was detected at specified times using a fluorometer (FLUOstar Galaxy).

\section{Immunoblotting}

Pelleted neutrophils infected with M.tb or stimulated with CoMTB were mixed with SDS lysis buffer. The samples were run on the NuPAGE ${ }^{\circledR} 4-12 \%$ Bis-Tris gels with SDS Running buffer (Invitrogen). Protein was transferred onto a nitrocellulose membrane (GE Healthcare). Primary antibody was diluted in 5\% BSA/0.1\% Tween and incubated overnight at $4{ }^{\circ} \mathrm{C}$ with agitation. Secondary antibody was added diluted in blocking buffer. Luminescence was demonstrated with ECL Substrate Reagent (Amersham Science) according to manufacturer's instructions and exposing the membrane to Hyperfilm ECL. Densitometric analysis was performed using Image $1.43 \mathrm{U}$ (NIH, USA).

\section{Real-time PCR}

Total RNA was extracted from $2 \times 10^{6}$ neutrophils using the RNeasy Mini Kit (Qiagen). Quantitative real-time RTPCR was performed using the One-Step RT-PCR Master Mix (Qiagen) according to manufacturer's instruction on a Stratagene Mx3000P platform using 5-10 $\mu \mathrm{g}$ per sample. MMP-9 (forward primer 5'-AGGCGCTCATGTAC CCTATGTAC-3', reverse primer 5'-GCCGTGGCT CAGGTTCA-3', Probe 5'-FAM-CATCCGGCACCTCT ATGGTCCTCG-TAMRA-3') with glyceraldehyde 3phosphate dehydrogenase (GAPDH) (Forward primer 5'-CGCTTCGCTCTCTGCTCCT-3', reverse primer $5^{\prime}$-CGACCAAATCCGTTGACTCC-3', probe 5'-HEXCGTCGCCAGCCGAGCCACAT-TAMRA-3') was analyzed in parallel. To accurately determine the quantitative change in RNA, standard curves were prepared from plasmids subjected to real-time PCR as above. MMP-9 data were normalized to GAPDH detected in the same sample.

\section{Immunofluorescence microscopy}

Permanox chamber slides (Nunc Lab-Tek) were coated with $25 \mu \mathrm{g} / \mathrm{ml}$ of DQ collagen for $30 \mathrm{~min}$. The samples were then fixed with $4 \%$ paraformaldehyde for $30 \mathrm{~min}$ and permeabilized with $0.5 \%$ saponin for $10 \mathrm{~min}$. The cells were washed before blocking with $10 \%$ human $\mathrm{AB}$ serum with $2.5 \%$ BSA and $0.05 \%$ saponin. Primary antibodies were added overnight. Chamber slides were washed prior to the addition of secondary antibodies. The chambers were subsequently removed from the slide, and saline was added. Images were captured using Leica confocal microscope (Leica TCS SP5) and processed using Leica LAS AF Lite 2.6.0 (Leica Microsystems, Germany) and Image $1.43 \mathrm{U}$ (NIH, USA).

\section{Flow cytometry}

Cell viability was assessed by staining neutrophils with annexin $\mathrm{V}$ and propidium iodide using Annexin $\mathrm{V}$ FITC Apoptosis Detection Kit (eBioscience, Affymetrix, California, USA) and LIVE/DEAD Fixable Stain Kit 
(Invitrogen). Neutrophils were stimulated with $200 \mathrm{ng} / \mathrm{ml}$ staurosporine to induce apoptosis, and this was used as a positive control for all experiments. Annexin V was detected on the FL-1 channel with propidium iodide and LIVE/DEAD Fixable Dead Cell Stain Kit on FL-3. A total of 50,000 events were gated and analyzed on BD FACSCalibur flow cytometer using CellQuest. Data was analyzed using FlowJo 7.6.5 (Tree Star).

\section{Statistical analyses}

Data were analyzed using GraphPad Prism (version 5.04, GraphPad Software). Data are expressed as mean \pm s.d. unless stated otherwise. All experiments are performed in biological triplicates on at least two separate occasions. Multiple intervention experiments are compared with one-way ANOVA followed by Tukey's post-test correction, while continuous variables between two sets of data are assessed using two-tailed Mann-Whitney $U$ test. Spearman's rank correlation tests are used for correlation analyses. $P$ values of less than 0.05 are taken as statistically significant.

\section{Results}

Neutrophils express MMP-9 in response to direct M.tb infection or stimulation by M.tb-driven monocyte-dependent networks and in patients with CNS-TB

First, we investigated MMP-9 secretion from primary human neutrophils in TB. Neutrophil MMP-9 secretion increased over $4 \mathrm{~h}$ in response to $M . t b$ infection (Fig. 1a, $P<0.0001$ ). By $24 \mathrm{~h}$, more than $80 \%$ of neutrophils were non-viable after infection by flow cytometry staining of annexin $\mathrm{V}$ and propidium iodide. Intercellular networks are crucial in TB [18], so we evaluated the effect of cross talk between neutrophils and monocytes [19] by using conditioned media from monocytes infected by M.tb (CoMTB). CoMTB stimulation caused significant upregulation of MMP-9 secretion by 3.5-fold compared to CoMCont (Fig. 1b, $P<0.0001$ ). CoMTB increased neutrophil MMP-9 gene expression 8.6-fold (Fig. 1c, $P<0.001$ ). As direct M.tb infection and intercellular network interactions occur simultaneously in $\mathrm{TB}$, we investigated the effect of concurrent M.tb and CoMTB stimulation of neutrophils and demonstrated an additive effect on MMP9 secretion (Fig. 1d). We also analyzed the secretion of NGAL which can restrict the growth of M.tb [20]. Neutrophil MMP-9 secretion closely correlated with the secretion of NGAL in response to both M.tb and CoMTB stimulation (Fig. 1e; $r=0.9, P<0.0001$ ).

To determine whether neutrophils express MMP-9 at the site of disease in patients with CNS-TB, we analyzed specimens from patients who underwent diagnostic biopsies. Polymorphonuclear neutrophils were observed around the granuloma on $\mathrm{H} \& \mathrm{E}$ staining (Fig. 1f). Neutrophil accumulation was confirmed by specific positive staining for neutrophil elastase and MMP-9 (Fig. 1g, h). MMP-9 was also seen in the blood vessel wall and perivascular interstitium (Fig. 1j). The M.tb-infected brain tissue with secondary antibody alone showed no immunoreactivity (Fig. 1k).

\section{M.tb-infected neutrophils degrade basement membrane matrix}

Type IV collagen is the main extracellular matrix fibril in the basement membrane of the BBB [21]. Therefore, we assessed the functional activity of neutrophil MMP-9 on this key structural protein. Confocal microscopy demonstrated type IV collagen degradation at the neutrophilcollagen interface in M.tb-infected neutrophils, but not uninfected neutrophils (Fig. 2a). Increased collagenase activity after $M . t b$ infection was confirmed by a quantitative fluorescence assay (Fig. 2b). Pre-treatment of M.tb-stimulated neutrophil supernatants with neutralizing antiMMP-9 antibody caused a dose-dependent inhibition of collagenase activity with a maximal effect at $10 \mu \mathrm{g} / \mathrm{ml}$ (Fig. $2 \mathrm{~b}, P<0.01$ ), demonstrating that this collagenolysis was MMP-9 dependent.

\section{Neutrophil MAP-kinase pathways regulate CoMTB-induced MMP-9 secretion}

Next, we investigated the neutrophil intracellular pathways regulating MMP-9 secretion. As the MAP-kinase pathway regulates MMPs in other CNS cell types [22], we evaluated components of the pathway following CoMTB stimulation of neutrophils. Immunoblotting revealed phosphorylation of p38, ERK, and JNK with corresponding increase of phospho-kinase to total kinase ratio after CoMTB stimulation (Fig. 3a-d). Using targeted inhibitors against p38 and ERK [23], we demonstrated a 48\% MMP9 inhibition with $10 \mu \mathrm{M}$ of p38 inhibitor SB203580 (Fig. 3e, $P<0.001$ ) and a $15 \%$ inhibition with $10 \mu \mathrm{M}$ of ERK inhibitor PD98059 compared to neutrophil stimulation with CoMTB alone (Fig. 3f, $P<0.05$ ). With live virulent $M . t b$ stimulation, we demonstrated phosphorylation of the p38 and ERK components of the MAP kinase pathway (Fig. 3g-i). However, in contrast to CoMTB stimulation, pre-incubation of neutrophils with p38 inhibitor or ERK inhibitors did not suppress MMP-9 secretion after M.tb infection (Fig. 3j, k).

The Akt pathway is activated and regulates MMP-9 secretion after CoMTB stimulation but not direct M.tb infection The $\mathrm{PI}_{3}$ kinase-Akt pathway regulates bronchial epithelial cell-derived MMPs in TB [24]. We had previously shown that Akt was phosphorylated in the presence of CoMTB [6] and therefore investigated if this path also regulates neutrophil MMP-9 secretion. Pre-treating neutrophils with $\mathrm{PI}_{3}$ kinase inhibitor LY294002 showed a dose-dependent suppression of MMP-9 of 1.6-fold with 


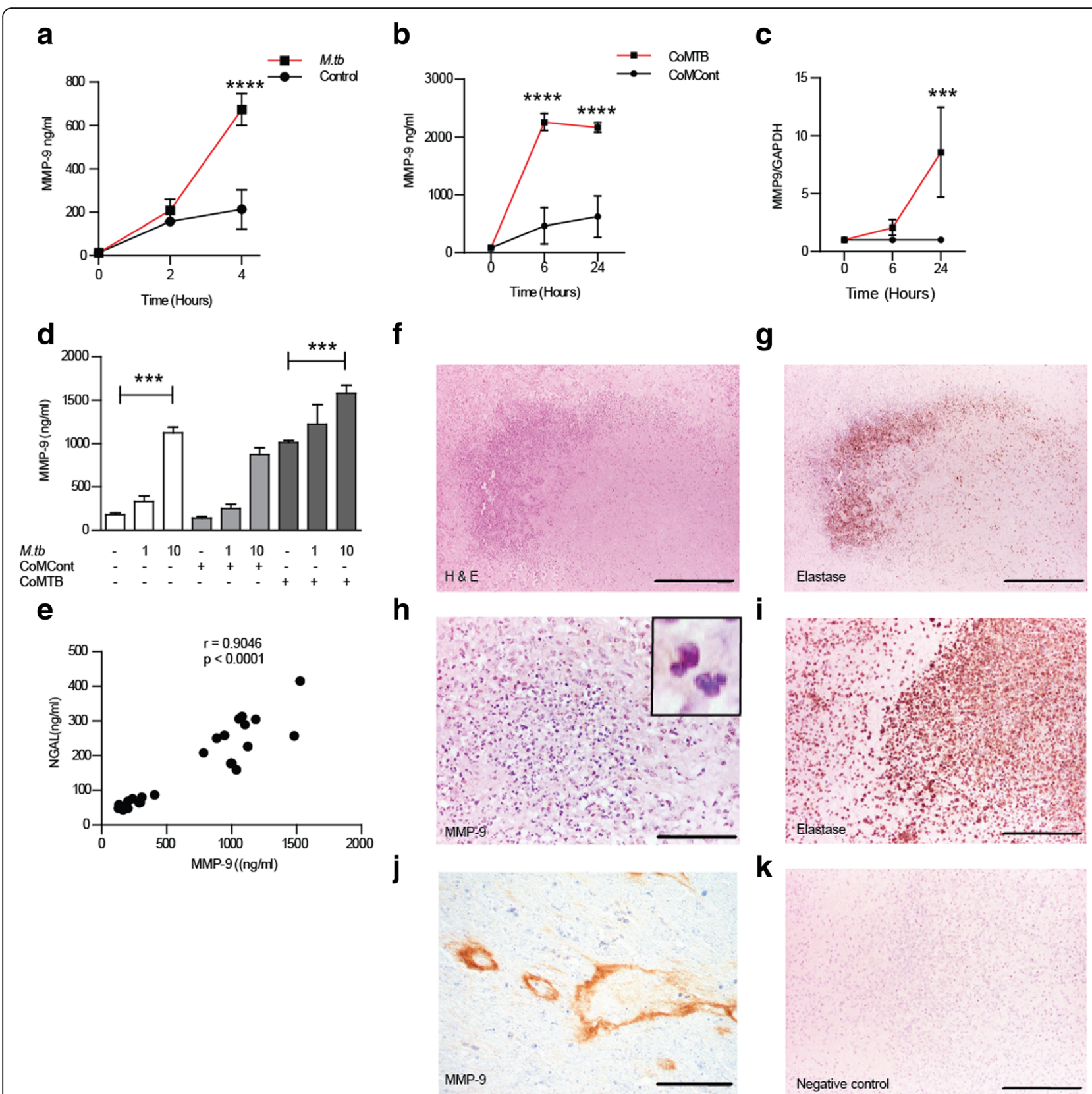

Fig. 1 Neutrophils secrete MMP-9 and are present in CNS-TB. a M.tb infection with MOI of 1 upregulates neutrophil MMP-9 secretion. b Neutrophils from healthy volunteers secrete MMP-9 in response to CoMTB. c Kinetics of Neutrophil MMP-9 gene expression after CoMTB stimulation. $\mathbf{d}$ M.tb and CoMTB stimulation have an additive effect on neutrophil MMP-9 secretion. e NGAL and MMP-9 concentrations closely correlate despite being located in different neutrophil granules. $\mathbf{f}-\mathbf{h}$ Neutrophils expressing MMP-9 are present in CNS TB granulomas and present in vessel walls and perivascular interstitial space. f H\&E, scale bar $250 \mu \mathrm{m}$. g Elastase immunoperoxidase, scale bar $250 \mu \mathrm{m}$ and $\mathbf{h}$ MMP-9 immunoperoxidase, $\times 20$ magnification, scale bar $50 \mu \mathrm{m}$. Inset shows neutrophils within lesions $(\times 40)$. Positive control on acute appendicitis with $\mathbf{i}$ elastase, scale bar $50 \mu \mathrm{m}$ and j MMP-9 on blood vessel wall and interstitium, scale bar 200 mm. k Negative control with omission of primary antibodies, scale bar represents $100 \mu \mathrm{m}$. Bars represent mean \pm s.d and represent at least two independent experiments performed in triplicate. ${ }^{*} P<0.05 ;{ }^{* *} P<0.01$; ${ }^{* *} P<0.001 ; * * * P<0.0001$

CoMTB stimulation (Fig. 4a, $P<0.01$ ). Similarly with M.tb, Akt was phosphorylated, indicating activation (Fig. 4b). However, $\mathrm{PI}_{3}$ kinase inhibitor LY294002 did not inhibit neutrophil MMP-9 secretion with M.tb stimulation (Fig. 4c).

\section{Transcription factor NF-kB regulates neutrophil MMP-9} in TB

NF-kB is one of the crucial transcription factors driving inflammation in TB [6]. We evaluated if NF-kB regulated neutrophil MMP-9 secretion by using NF-kB p65 


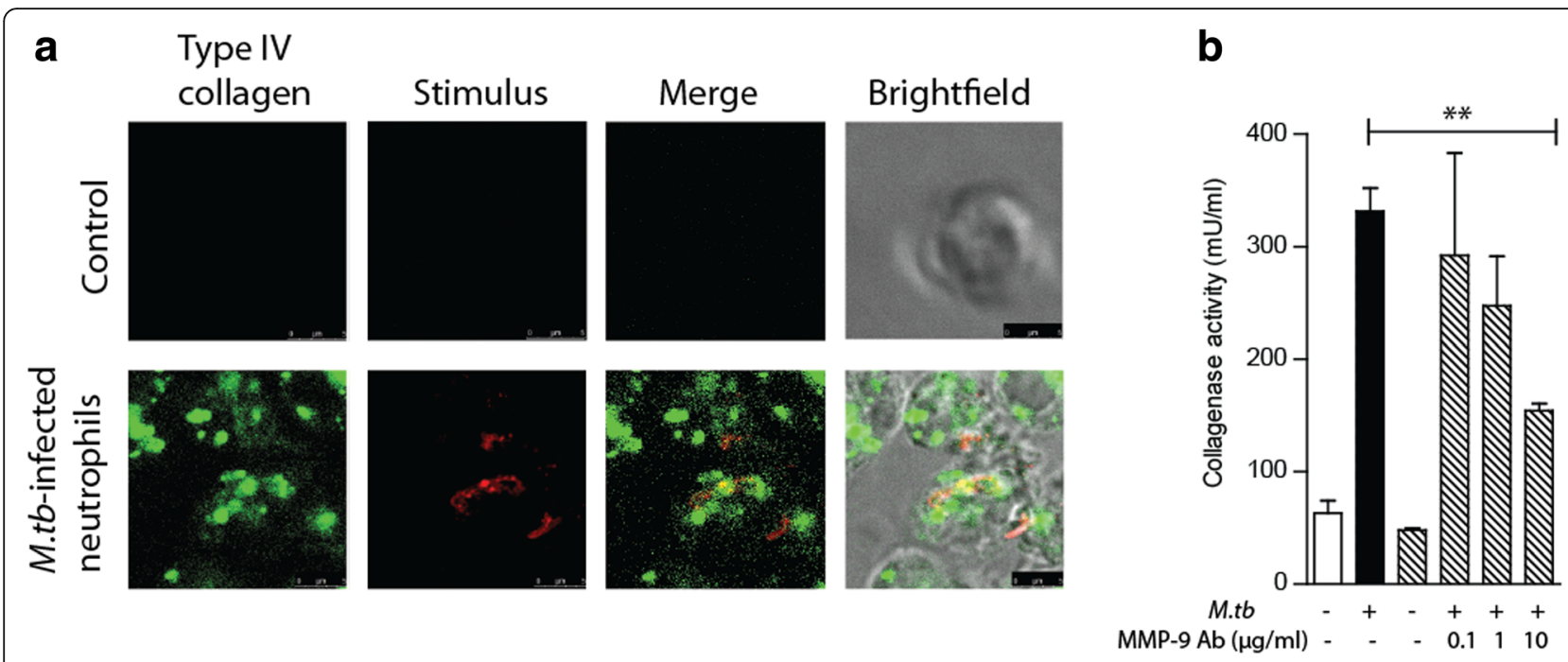

Fig. 2 Collagen is degraded by neutrophils stimulated with M.tb in an MMP-9 dependent manner. a Confocal microscopy of Type IV DQ collagen coated slides demonstrating collagen degradation in the presence of M.tb stimulation. DQ collagen fluorescence increases after degradation. $\mathbf{b}$ Type IV collagenase activity is inhibited by MMP-9 neutralizing antibody. Bars represent mean \pm s.d and represent two independent experiment performed in triplicate. ${ }^{*} P<0.01$

subunit inhibitor helenalin $\left(\mathrm{IC}_{50} 10-50 \mu \mathrm{M}\right)$ and $\mathrm{IKK}_{2}$ inhibitor SC-514 $\left(\mathrm{IC}_{50} 3-12 \mu \mathrm{M}\right)$. In the presence of CoMTB stimulation, we demonstrated a dose-dependent suppression of neutrophil MMP-9 to baseline with helenalin (Fig. 5a, $P<0.001$ ). Suppression of neutrophil MMP-9 secretion was similarly achieved with SC-514 (Fig. 5b, $P<0.01$ ). Pre-treating neutrophils with helenalin before M.tb infection also resulted in a decrease in neutrophil MMP-9 secretion, which was maximal at a dose of $100 \mu \mathrm{M}$ (Fig. 5c, $P<0.001$ ), but at higher concentrations, neutrophil viability declined markedly to less than $70 \%$ by FACS. Therefore, NF-kB regulates neutrophil MMP-9 secretion for both direct infection and intercellular networks.

\section{Anti-TNFa suppresses neutrophil MMP-9 secretion, but dexamethasone does not}

Neutrophil MMPs are raised in CNS-TB [8], and antiTNF $\alpha$ antagonists have been used to treat severe paradoxical reaction in CNS-TB with improvement to neurological outcome $[25,26]$. We investigated the effect of TNF $\alpha$ neutralizing antibodies on neutrophil MMP secretion. Pre-incubating CoMTB with anti-TNF $\alpha$ prior to stimulating human neutrophils resulted in suppression of MMP-9 secretion and gene expression which was maximal at a dose of $5 \mu \mathrm{g} / \mathrm{ml}$ (Fig. 6a, b; $P<0.01$ and $P<0.001$, respectively). TIMP-1 secretion was unaffected by anti-TNF $\alpha$ (Fig. 6c) while TIMP-2 secretion was slightly suppressed in a dose-dependent manner (Fig. 6d, $P<0.01$ ). Dexamethasone is an established adjunct treatment for patients with CNSTB that improves mortality but does not significantly affecting neurological outcome [1], so we investigated the effect of dexamethasone on neutrophil MMP secretion. MMP-9 secretion was unaffected by dexamethasone at a dose of $5 \mu \mathrm{g} / \mathrm{ml}$, similar to the dose used in clinical trials (Fig. 6e). However, CoMTBdriven neutrophil MMP-8 secretion was inhibited by $32 \%$ (Fig. 6f, $P<0.001$ ). We also evaluated if TNF $\alpha$ drove neutrophil MMP-9 secretion in M.tb infection, but this was not altered.

\section{Discussion}

CNS-TB is a devastating infection driven by an excessive host immune response to infection and results in neurological morbidity and death. Our findings reveal a mechanism by which neutrophils contribute to tissue destruction in the brain, further supporting the role of the granulocyte in CNS-TB pathogenesis $[8,9,27]$. We have demonstrated that neutrophils within granulomas express MMP-9 in the brain biopsies from patients with proven CNS-TB, further highlighting the role of MMPs in pathology of TB granulomas [28]. Neutrophils secrete MMP-9 in response to both direct M.tb infection and monocyte-dependent networks in CNS-TB. The combination of the two stimuli has an additive effect to MMP-9 secretion, which is partially driven by TNF $\alpha$, as demonstrated by a decrease in MMP-9 with the addition of neutralizing anti-TNF $\alpha$ antibodies in CoMTB stimulation. The secretion of MMP-9 from neutrophils results in the destruction of type IV collagen, a major constituent of the basement membrane in the BBB, and this was inhibited by neutralizing MMP-9. This observation may explain why increased MMP-9 expression in CNS 
a

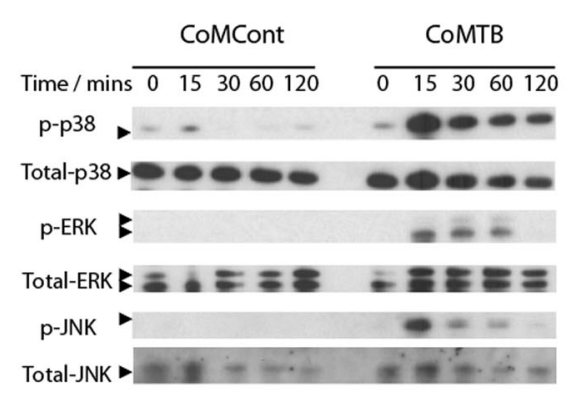

C
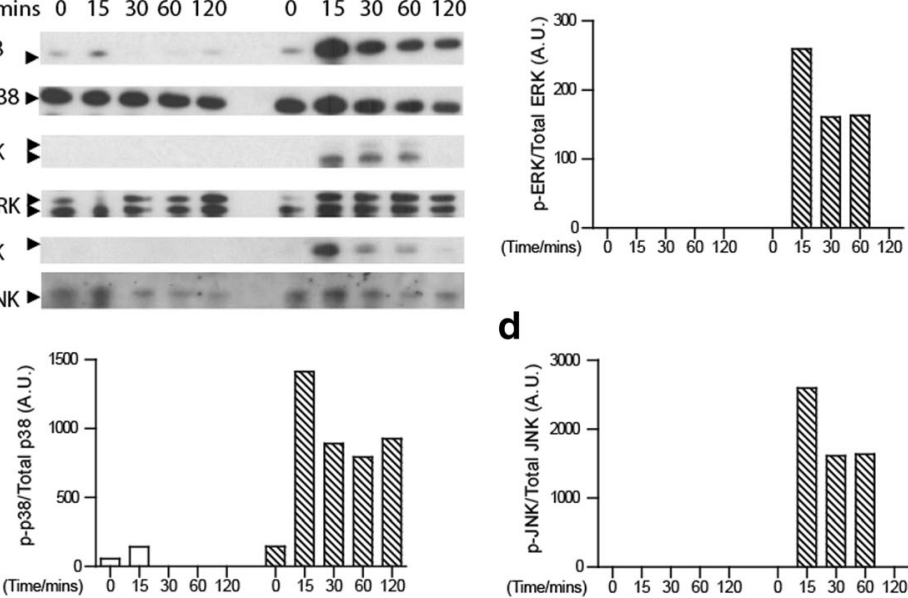

d

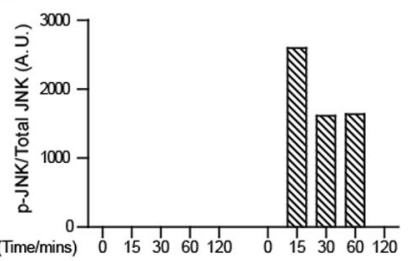

e

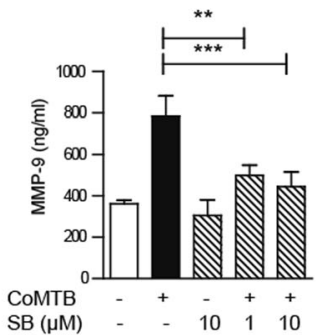

f

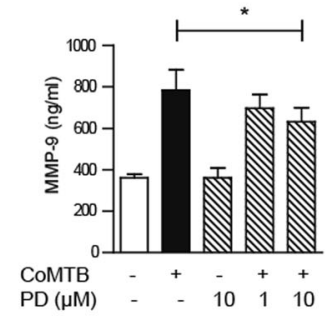

g

Time/mins $\frac{\text { Control }}{0153060120} \quad \frac{\text { M.tb }}{0153060120}$

Time/mins $\frac{\text { Control }}{0153060120} \quad \frac{\text { M.tb }}{0153060120}$

h
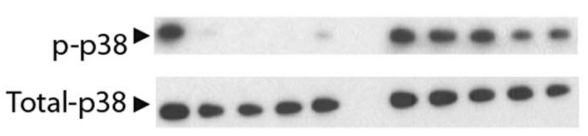

p-ERK

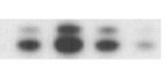

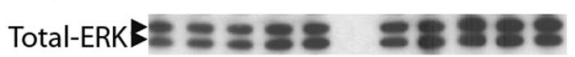

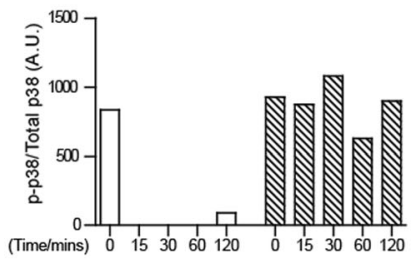

i

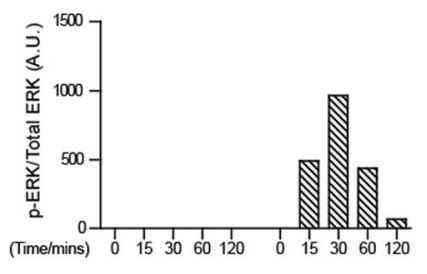

j

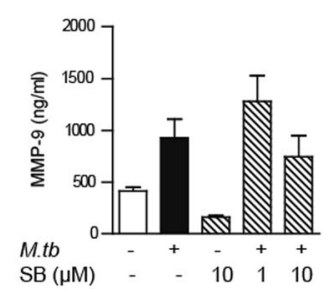

k

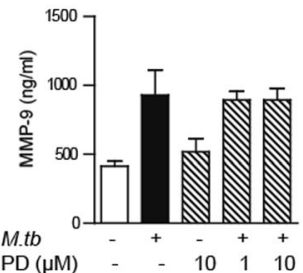

Fig. 3 (See legend on next page.) 
(See figure on previous page.)

Fig. 3 MAP-kinase pathways are activated in neutrophils in TB and regulate MMP-9 secretion. a Representative immunoblots of p-38, ERK, and JNK MAP kinases in neutrophils stimulated with COMTB. COMTB increases phosphorylation of all pathways from 15 min. Densitometric analysis of response to CoMCont and CoMTB stimulation of $\mathbf{b}$ phospho-p38 to total p38, c phospho-ERK to total ERK, and $\mathbf{d}$ phospho-JNK to total JNK. e CoMTB-stimulated MMP-9 secretion is suppressed by p-38 inhibitor SB203580. f MMP-9 secretion with CoMTB is inhibited by ERK inhibitor PD98059. g Representative immunoblots to detect p-38, ERK MAP kinases in neutrophils stimulated with M.tb. Densitometric analysis of response to control (PBS) and M.tb infection of $\mathbf{h}$ phospho-p-38 to total p-38 and $\mathbf{i}$ phospho-JNK to total JNK. $\mathbf{j}$ Pre-incubation with p-38 inhibitor SB does not suppress MMP-9 secretion in M.tb-infected neutrophils. $\mathbf{k}$ Pre-incubation with ERK inhibitor PD does not affect MMP-9 secretion by M.tb-stimulated neutrophils. All chemical inhibitor experiments were performed by pre-incubating neutrophils for 30 min before stimulating for $4 \mathrm{~h}$. Bars represent mean \pm s.d and are representative of at least two independent experiments performed in triplicate. ${ }^{*} P<0.05 ;{ }^{* *} P<0.01$; ${ }^{* * *} P<0.001$

diseases corresponds to BBB disruption and is associated with neurological deficit and death [13, 29]. There are several potential targets in the MMP-9 regulatory pathway for host-directed therapy in CNS-TB, and of note, both specific and broad-based MMP-inhibitors are currently being explored as adjunctive therapy in other CNS-diseases such as autoimmune encephalomyelitis and stroke [30, 31].

We explored potential intracellular switch points in the regulation of neutrophil MMP-9 secretion and showed that the MAP-kinase, Akt-PI ${ }_{3}$ kinase, and NF-kB have the key roles. This is consistent with our findings of MMP regulation in other CNS cells such as astrocytes and microglial cells [32-34]. However, we observed divergent effects with M.tb and CoMTB stimulation on neutrophil intracellular signaling pathways. In the presence of virulent M.tb, neutrophil MMP-9 secretion was not suppressed by targeted chemical inhibitors of intracellular signaling paths upstream of transcription. In contrast, CoMTB, which contains cytokines including TNFo, results in neutophil MMP-9 secretion and this secretion was decreased by blockade of the p38 and ERK MAP kinases and PI3 kinase paths. This was in contrast to previous findings that TNF $\alpha$-induced neutrophil MMP-9 secretion is independent of the p38 and ERK kinases [16]. We postulate the differences caused by CoMTB and M.tb may in part be due to M.tb triggering cellular necrosis in neutrophils that we and others demonstrated [35], leading to unregulated release of their proteases, and consequently, pathway modulation did not affect total MMP-9 release. This highlights the importance of monocytes in mediating neutrophil secretion of MMP-9 that drives matrix destruction in TB.

NF-kB inhibition decreased neutrophil MMP-9 secretion in response to both direct infection and monocytedependent networks. We have previously demonstrated

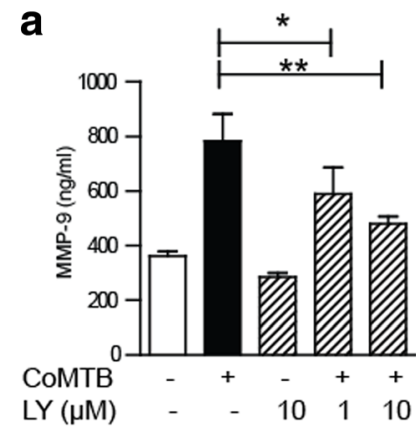

C

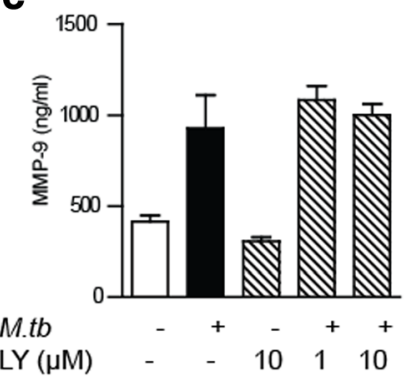

b
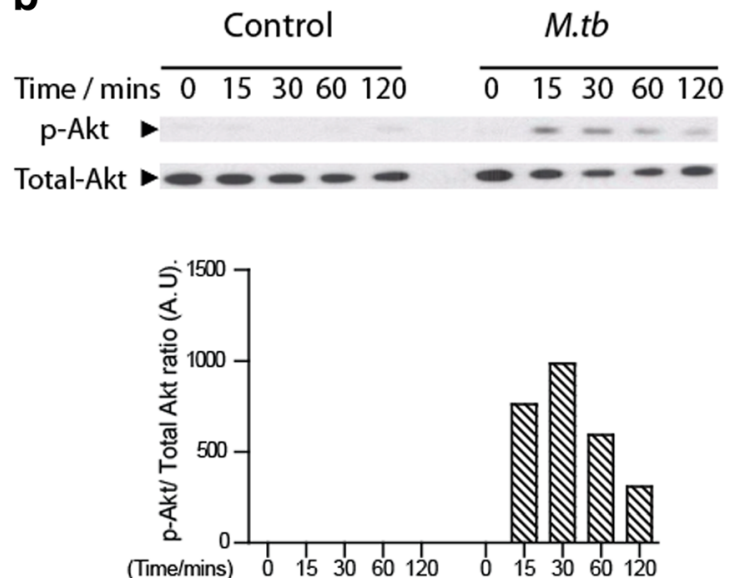

Fig. 4 The Akt pathway regulates neutrophil MMP-9 secretion in TB. a Pre-incubation with PI3-kinase inhibitor LY294002 suppresses MMP-9 secretion with COMTB. b Representative immunoblots to detect p-Akt in neutrophils infected with M.tb show increased phosphorylation with corresponding densitometric analysis. c Pre-incubation with PI3-kinase inhibitor LY does not affect MMP-9 secretion after M.tb infection. Bars represent mean \pm s.d and are representative of at least two independent experiments performed in triplicate. ${ }^{*} P<0.05 ;{ }^{*} P<0.01$ 


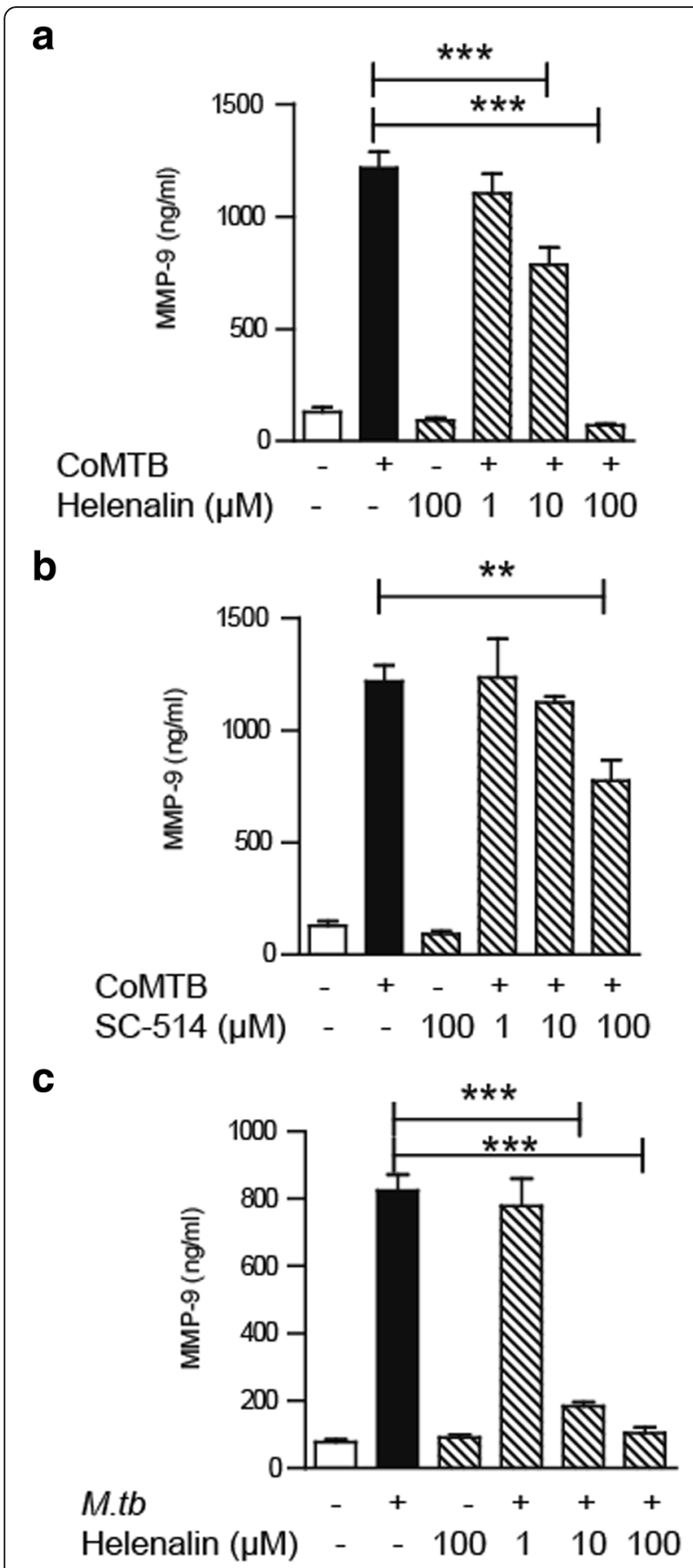

Fig. 5 Neutrophil MMP-9 secretion is regulated by NF-kB. a Preincubation with helenalin inhibits MMP-9 secretion after COMTB stimulation. b Pre-incubation with IKK2 inhibitor SC-514 inhibits MMP-9 secretion with COMTB stimulation. c Pre-incubation with NF-kB inhibitor helenalin inhibits MMP-9 secretion after M.tb infection. Bars represent mean \pm s.d and are representative of at least two independent experiments performed in triplicate. ${ }^{* *} P<0.01$; ${ }^{* *} P<0.001$

that NF-kB inhibition decreased neutrophil MMP-8 secretion which drove type I collagen degradation [6], a fibril which is important in lung TB but minimally present in the central nervous system. We now demonstrate that neutrophil MMP-9, which drives destruction of type IV collagen present in the BBB, is suppressed by NF-kB inhibition. Thalidomide inhibits NF-kB by suppressing IkB kinase activity [36] and has been used to treat paradoxical reaction in CNS-TB [37, 38], but its considerable side effects such as teratogenicity and peripheral neuropathy mean that its potential as use for a host-directed therapy will be very limited.

Finally, we investigated the effects of anti-TNF $\alpha$ and dexamethasone on neutrophil MMP-9 secretion and found that anti-TNF $\alpha$ treatment significantly inhibited neutrophil MMP-9 secretion, while dexamethasone partially inhibited MMP-8 but did not affect MMP-9 secretion. The lack of response of neutrophil MMPs to dexamethasone was unexpected, given our previous finding that dexamethasone decreased CSF MMP-9 in a cohort of Vietnamese patients with CNS-TB [8]. The cells other than neutrophils such as the astrocytes, microglial, and neuronal cells also secrete MMP-9 and may contribute to the total suppression of CSF MMP-9 causing the effect that we previously observed. Glucocorticoids are established adjuncts to treatment of CNS$\mathrm{TB}$ and inhibit NF-kB activity through induction of IkB synthesis, among other mechanisms, to decrease inflammatory responses and decrease MMP-9 [39]. However, steroids have significant off-target effects such as immunosuppression, steroid-induced diabetes mellitus, and Cushing's syndrome. Anti-TNF $\alpha$ inhibitors are sometimes used to treat refractory paradoxical reactions in CNS-TB $[25,26]$ and may lead to improved neurological outcome by decreasing MMP9 secretion.

Our study has limitations, including the use of chemical inhibitors, which may have off-target effects, to evaluate intracellular signaling pathways in neutrophils. Ideally, selective pathway inhibition using siRNA would be more specific. However, this is technically challenging in primary neutrophils as they die rapidly in vitro and hence, we and others have been unable to proceed with this approach. Also, our findings of TNF $\alpha$ in mediating neutrophil MMP secretion have to be carefully interpreted in clinical care as there is a potential optimal concentration for host control of infection [18]. While excessive TNF $\alpha$ drives paradoxical TB reactions, blocking TNF $\alpha$ can lead to reactivation of latent $\mathrm{TB}[15,40]$, so any therapeutic intervention requires very careful evaluation.

\section{Conclusions}

In summary, we show MMP-9 is expressed by neutrophils during the host response in CNS-TB. Neutrophil MMP-9 gene expression and secretion are driven by 
a

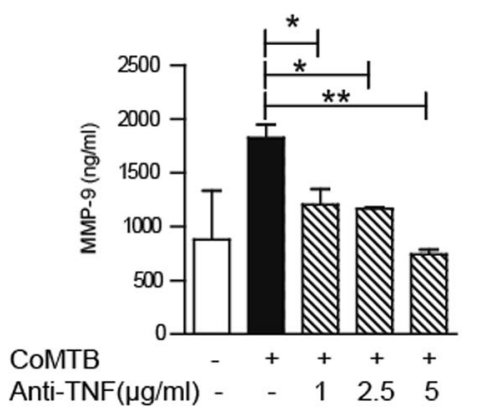

C

CoMTB

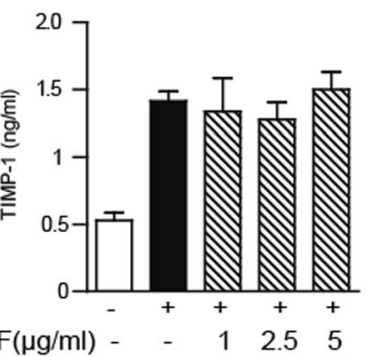

e

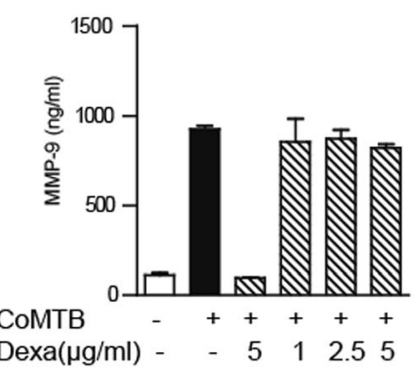

b

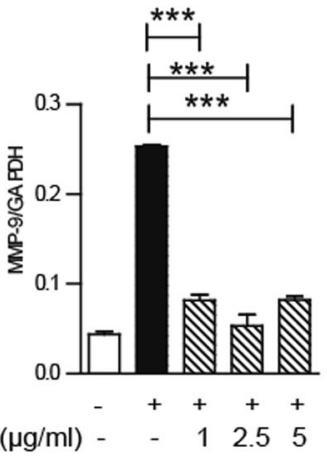

d

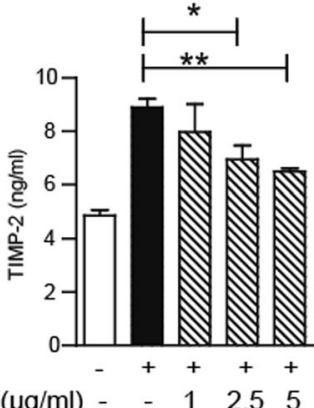

Anti-TNF $(\mu \mathrm{g} / \mathrm{ml})-\quad-12.55$

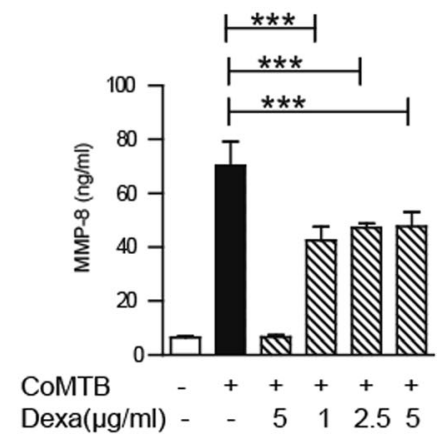

Fig. 6 Anti-TNFa suppresses neutrophil MMP-9 secretion, but dexamethasone does not. a MMP-9 secretion is inhibited by anti-TNFa pre-treatment of neutrophils. CoMTB was incubated with anti-TNFa antibodies for $1 \mathrm{~h}$ before neutrophil stimulation. $\mathbf{b}$ Anti-TNFa inhibits MMP-9 gene expression. $\mathbf{c}$ TIMP-1 secretion is not affected by anti-TNFa. $\mathbf{d}$ TIMP-2 secretion is reduced by anti-TNFa. e MMP-9 secretion is not affected by dexamethasone. $\mathbf{f}$ Dexamethasone inhibits neutrophil MMP-8 secretion. Bars represent mean \pm s.d and are representative of at least two independent experiments performed in triplicate. ${ }^{*} P<0.05 ;{ }^{* *} P<0.01 ;{ }^{* *} P<0.001$

both direct effects of M.tb infection and monocytedependent networks. Destruction of type IV collagen, a major component of the $\mathrm{BBB}$, is driven by neutrophil MMP-9. The MAP-kinase and Akt-PI 3 kinase pathways regulate MMP-9 secretion through monocyte-dependent networks but not after direct $M . t b$ infection. Neutralizing TNF $\alpha$ significantly suppressed neutrophil MMP-9 secretion and gene expression. Host-directed therapies that target MMPs and the key regulatory pathways should be evaluated to improve neurological and mortality outcomes in CNS-TB.

\section{Abbreviations}

BBB: Blood-brain barrier; CNS-TB: Central nervous system tuberculosis; COMTB: Conditioned media of monocytes infected with M. tuberculosis; CSF: Cerebrospinal fluid; HIV: Human immunodeficiency virus;
LTA : Leukotriene A4; M.tb: Mycobacterium tuberculosis; MAP-kinase: Mitogenactivated protein kinase; MMP: Matrix metalloproteinases; NF-kB: Nuclear factor-kB; NGAL: Neutrophil gelatinase-associated lipocalin; PCR: Polymerase chain reaction; TB: Tuberculosis; TIMP: Tissue inhibitor of metalloproteinases; TNF: Tumor necrosis factor

\section{Acknowledgements}

Tissue samples and associated clinical and neuropathological data were supplied by the Parkinson's UK Brain Bank, funded by Parkinson's UK, a charity registered in England and Wales (258197) and in Scotland (SC037554). We thank the UK Parkinson's Tissue Bank at Imperial College for supplying the samples of the normal brain tissue and donors and their families for making this project possible.

\section{Funding}

C.W.M.O. is funded by the Singapore National Medical Research Council on an NRF-MOH Healthcare Research Scholarship. J.S.F. and P.E. acknowledge support of the NIHR Biomedical Research Centre at Imperial College. 


\section{Availability of data and materials}

Not applicable.

\section{Authors' contributions}

JSF conceived the project. CWMO, PTE, and JSF designed the experiments and analyzed the data. CWMO and PJP performed the experiments and generated the data. CWMO recruited the healthy volunteers. FR generated the immunohistochemistry data. CWMO, PTE, and JSF wrote the manuscript which was reviewed by all authors. All authors read and approved the final manuscript.

\section{Competing interests}

The authors declare that they have no competing interests.

\section{Consent for publication}

Not applicable.

\section{Ethics approval and consent to participate}

For extraction of the primary human neutrophils, ethical approval and consent for obtaining healthy human blood volunteer was provided by the Outer West London Research Ethics Committee and written informed consent was obtained from individuals. The CNS-TB brain biopsy specimens were provided by the Imperial College Healthcare Tissue Bank. Ethical permission for this study was obtained from the Hammersmith Hospitals Research Ethics Committee.

\section{Author details}

${ }^{1}$ Section of Infectious Diseases and Immunity, Hammersmith Campus, Imperial College London, 8th floor Commonwealth Building, Du Cane Road, London W12 ONN, UK. ${ }^{2}$ Division of Infectious Diseases, Department of Medicine, Yong Loo Lin School of Medicine, National University of Singapore, Singapore, Singapore. ${ }^{3}$ Department of Histopathology, Hammersmith Campus, Imperial College London, London, UK. ${ }^{4}$ Division of Neuroscience and Experimental Psychology, University of Manchester, Manchester, UK. ${ }^{5} \mathrm{NIHR}$ Respiratory Biomedical Research Unit, Faculty of Medicine, University of Southampton, Southampton, UK.

Received: 19 September 2016 Accepted: 23 January 2017

Published online: 07 February 2017

\section{References}

1. Thwaites GE, Nguyen DB, Nguyen HD, Hoang TQ, Do TT, Nguyen TC, Nguyen QH, Nguyen TT, Nguyen NH, Nguyen TN, et al. Dexamethasone for the treatment of tuberculous meningitis in adolescents and adults. N Engl J Med. 2004;351:1741-51.

2. Torok ME, Nguyen DB, Tran TH, Nguyen TB, Thwaites GE, Hoang TQ, Nguyen HD, Nguyen TC, Hoang HT, Wolbers M, Farrar JJ. Dexamethasone and long-term outcome of tuberculous meningitis in Vietnamese adults and adolescents. PLoS One. 2011;6, e27821.

3. Tobin DM, Roca FJ, Oh SF, McFarland R, Vickery TW, Ray JP, Ko DC, Zou Y, Bang ND, Chau TT, et al. Host genotype-specific therapies can optimize the inflammatory response to mycobacterial infections. Cell. 2012;148:434-46.

4. Wallis RS, Hafner R. Advancing host-directed therapy for tuberculosis. Nat Rev Immunol. 2015;15:255-63.

5. Nouailles G, Dorhoi A, Koch M, Zerrahn J, Weiner J, 3rd, Fae KC, Arrey F, Kuhlmann S, Bandermann S, Loewe D, et al. CXCL5-secreting pulmonary epithelial cells drive destructive neutrophilic inflammation in tuberculosis. J Clin Invest. 2014;124(3):1268-82.

6. Ong CW, Elkington PT, Brilha S, Ugarte-Gil C, Tome-Esteban MT, Tezera LB, Pabisiak PJ, Moores RC, Sathyamoorthy T, Patel V, et al. Neutrophil-derived MMP-8 drives AMPK-dependent matrix destruction in human pulmonary tuberculosis. PLoS Pathog. 2015;11, e1004917.

7. Thwaites GE, Simmons CP, Than Ha Quyen N, Thi Hong Chau T, Phuong Mai P, Thi Dung N, Hoan Phu N, White NP, Tinh Hien T, Farrar JJ. Pathophysiology and prognosis in Vietnamese adults with tuberculous meningitis. J Infect Dis. 2003;188:1105-15.

8. Green JA, Tran CT, Farrar JJ, Nguyen MT, Nguyen PH, Dinh SX, Ho ND, Ly CV, Tran HT, Friedland JS, Thwaites GE. Dexamethasone, cerebrospinal fluid matrix metalloproteinase concentrations and clinical outcomes in tuberculous meningitis. PLoS One. 2009;4, e7277.
9. Marais S, Wilkinson KA, Lesosky M, Coussens AK, Deffur A, Pepper DJ, Schutz C, Ismail Z, Meintjes G, Wilkinson RJ. Neutrophil-associated central nervous system inflammation in tuberculous meningitis immune reconstitution inflammatory syndrome. Clin Infect Dis. 2014;59:1638-47.

10. Shi Y, Zhang L, Pu H, Mao L, Hu X, Jiang X, Xu N, Stetler RA, Zhang F, Liu X, et al. Rapid endothelial cytoskeletal reorganization enables early blood-brain barrier disruption and long-term ischaemic reperfusion brain injury. Nat Commun. 2016;7:10523.

11. Valentin-Torres A, Savarin C, Hinton DR, Phares TW, Bergmann CC, Stohlman SA. Sustained TNF production by central nervous system infiltrating macrophages promotes progressive autoimmune encephalomyelitis. $J$ Neuroinflammation. 2016;13:46.

12. Lukes A, Mun-Bryce S, Lukes M, Rosenberg GA. Extracellular matrix degradation by metalloproteinases and central nervous system diseases. Mol Neurobiol. 1999;19:267-84.

13. Price NM, Farrar J, Tran $T$, Nguyen TH, Tran TH, Friedland JS. Identification of a matrix-degrading phenotype in human tuberculosis in vitro and in vivo. J Immunol. 2001;166:4223-30.

14. Roca FJ, Ramakrishnan L. TNF dually mediates resistance and susceptibility to mycobacteria via mitochondrial reactive oxygen species. Cell. 2013;153:521-34.

15. Keane J. Tumor necrosis factor blockers and reactivation of latent tuberculosis. Clin Infect Dis. 2004;39:300-2.

16. Chakrabarti S, Zee JM, Patel KD. Regulation of matrix metalloproteinase-9 (MMP-9) in TNF-stimulated neutrophils: novel pathways for tertiary granule release. J Leukoc Biol. 2006;79:214-22.

17. Elkington PT, Green JA, Emerson JE, Lopez-Pascua LD, Boyle JJ, O'Kane CM, Friedland JS. Synergistic up-regulation of epithelial cell matrix metalloproteinase-9 secretion in tuberculosis. Am J Respir Cell Mol Biol. 2007;37:431-7.

18. Ong CW, Elkington PT, Friedland JS. Tuberculosis, pulmonary cavitation, and matrix metalloproteinases. Am J Respir Crit Care Med. 2014;190:9-18.

19. Soehnlein O, Zernecke A, Eriksson EE, Rothfuchs AG, Pham CT, Herwald H, Bidzhekov K, Rottenberg ME, Weber C, Lindbom L. Neutrophil secretion products pave the way for inflammatory monocytes. Blood. 2008;1 12:1461-71.

20. Martineau AR, Newton SM, Wilkinson KA, Kampmann B, Hall BM, Nawroly N, Packe GE, Davidson RN, Griffiths CJ, Wilkinson RJ. Neutrophil-mediated innate immune resistance to mycobacteria. J Clin Invest. 2007;117:1988-94.

21. Kuhn K. Basement membrane (type IV) collagen. Matrix Biol. 1995;14:439-45.

22. Green JA, Rand L, Moores R, Dholakia S, Pezas T, Elkington PT, Friedland JS. In an in vitro model of human tuberculosis, monocyte-microglial networks regulate matrix metalloproteinase- 1 and -3 gene expression and secretion via a p38 mitogen activated protein kinase-dependent pathway. J Neuroinflammation. 2013;10:107.

23. Syrbu SI, Waterman WH, Molski TF, Nagarkatti D, Hajjar JJ, Sha'afi RI. Phosphorylation of cytosolic phospholipase A2 and the release of arachidonic acid in human neutrophils. J Immunol. 1999;162:2334-40.

24. Singh S, Saraiva L, Elkington PT, Friedland JS. Regulation of matrix metalloproteinase-1, -3 , and -9 in Mycobacterium tuberculosis-dependent respiratory networks by the rapamycin-sensitive PI3K/p70(S6K) cascade. FASEB J. 2014;28:85-93.

25. Blackmore TK, Manning L, Taylor WJ, Wallis RS. Therapeutic use of infliximab in tuberculosis to control severe paradoxical reaction of the brain and lymph nodes. Clin Infect Dis. 2008;47:e83-5.

26. Wallis RS, van Vuuren C, Potgieter S. Adalimumab treatment of lifethreatening tuberculosis. Clin Infect Dis. 2009:48:1429-32.

27. Berry MP, Graham CM, McNab FW, Xu Z, Bloch SA, Oni T, Wilkinson KA, Banchereau R, Skinner J, Wilkinson RJ, et al. An interferon-inducible neutrophil-driven blood transcriptional signature in human tuberculosis. Nature. 2010;466:973-7.

28. Volkman HE, Pozos TC, Zheng J, Davis JM, Rawls JF, Ramakrishnan L. Tuberculous granuloma induction via interaction of a bacterial secreted protein with host epithelium. Science. 2010;327:466-9.

29. Seo JE, Hasan M, Han JS, Kang MJ, Jung BH, Kwok SK, Kim HY, Kwon OS. Experimental autoimmune encephalomyelitis and age-related correlations of NADPH oxidase, MMP-9, and cell adhesion molecules: the increased disease severity and blood-brain barrier permeability in middle-aged mice. J Neuroimmunol. 2015;287:43-53.

30. Yang Y, Salayandia VM, Thompson JF, Yang LY, Estrada EY. Attenuation of acute stroke injury in rat brain by minocycline promotes blood-brain barrier remodeling and alternative microglia/macrophage activation during recovery. J Neuroinflammation. 2015;12:26. 
31. Liechti FD, Bachtold F, Grandgirard D, Leppert D, Leib SL. The matrix metalloproteinase inhibitor RS-130830 attenuates brain injury in experimental pneumococcal meningitis. J Neuroinflammation. 2015;12:43.

32. Harris JE, Nuttall RK, Elkington PT, Green JA, Horncastle DE, Graeber MB, Edwards DR, Friedland JS. Monocyte-astrocyte networks regulate matrix metalloproteinase gene expression and secretion in central nervous system tuberculosis in vitro and in vivo. J Immunol. 2007;178:1199-207.

33. Green JA, Dholakia S, Janczar K, Ong CW, Moores R, Fry J, Elkington PT, Roncaroli F, Friedland JS. Mycobacterium tuberculosis-infected human monocytes down-regulate microglial MMP-2 secretion in CNS tuberculosis via TNFalpha, NFkappaB, p38 and caspase 8 dependent pathways. J Neuroinflammation. 2011;8:46.

34. Green JA, Elkington PT, Pennington CJ, Roncaroli F, Dholakia S, Moores RC, Bullen A, Porter JC, Agranoff D, Edwards DR, Friedland JS. Mycobacterium tuberculosis upregulates microglial matrix metalloproteinase-1 and -3 expression and secretion via NF-kappaB- and activator protein-1-dependent monocyte networks. J Immunol. 2010;184:6492-503.

35. Francis RJ, Butler RE, Stewart GR. Mycobacterium tuberculosis ESAT-6 is a leukocidin causing $\mathrm{Ca} 2+$ influx, necrosis and neutrophil extracellular trap formation. Cell Death Dis. 2014;5, e1474.

36. Keifer JA, Guttridge DC, Ashburner BP, Baldwin Jr AS. Inhibition of NF-kappa B activity by thalidomide through suppression of IkappaB kinase activity. J Biol Chem. 2001;276:22382-7.

37. van Toorn R, du Plessis AM, Schaaf HS, Buys H, Hewlett RH, Schoeman JF. Clinicoradiologic response of neurologic tuberculous mass lesions in children treated with thalidomide. Pediatr Infect Dis J. 2015;34:214-8.

38. van Toorn R, Rabie H, Dramowski A, Schoeman JF. Neurological manifestations of TB-IRIS: a report of 4 children. Eur J Paediatr Neurol. 2012; 16:676-82.

39. Auphan N, DiDonato JA, Rosette C, Helmberg A, Karin M. Immunosuppression by glucocorticoids: inhibition of NF-kappa B activity through induction of I kappa B synthesis. Science. 1995;270:286-90.

40. Marais S, Meintjes G, Pepper DJ, Dodd LE, Schutz C, Ismail Z, Wilkinson KA, Wilkinson RJ. Frequency, severity, and prediction of tuberculous meningitis immune reconstitution inflammatory syndrome. Clin Infect Dis. 2013;56:450-60.

\section{Submit your next manuscript to BioMed Central and we will help you at every step:}

- We accept pre-submission inquiries

- Our selector tool helps you to find the most relevant journal

- We provide round the clock customer support

- Convenient online submission

- Thorough peer review

- Inclusion in PubMed and all major indexing services

- Maximum visibility for your research

Submit your manuscript at www.biomedcentral.com/submit

) Biomed Central 